\title{
Herausragender Bestand zur Wirtschafts- und Kunstgeschichte
}

\author{
Bücher aus dem Landesgewerbeamt
}

Im August 1971 erfuhr die Württembergische Landesbibliothek einen erheblichen Bestandszuwachs durch die Übernahme von Bänden aus der Bibliothek des Landesgewerbeamts Stuttgart. Diese Verlagerung erhielt durch ein Verwaltungsabkommen zwischen dem Wirtschafts- und dem Kultusministerium Baden-Württembergs vom 12.9. bzW. 6.10.1972 eine rechtliche Bestätigung. Es handelte sich zunächst um ca. 24.600 Monographien in 27.000 Bänden sowie um 71 bis mindestens 1968 abonnierte Zeitschriften. Nach Abzug von Dubletten, die teilweise an andere staatliche Institutionen abgegeben wurden, konnten bis jetzt über 14.000 Monographien-Bände in der WLB katalogisiert und unter der speziellen Signaturgruppe A22 geschlossen aufgestellt werden. Die Zeitschriften-Bände wurden in die Jahresringe der Numerus-CurrensSignaturen eingearbeitet.

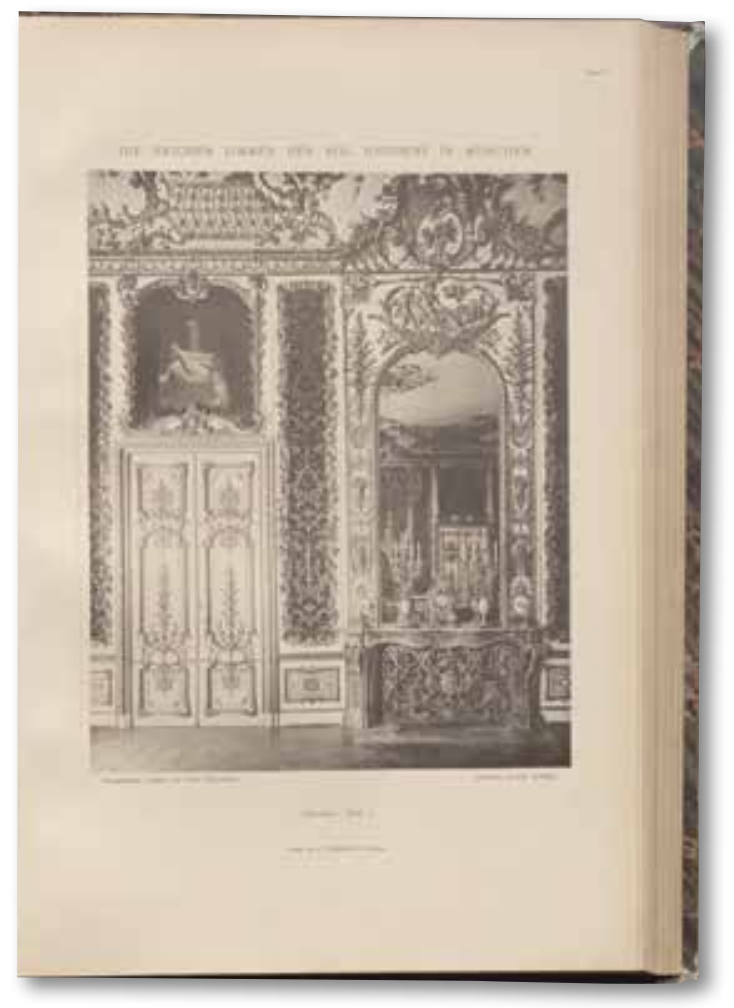

Abb. 1: Schloss Nymphenburg (A22g/357)

1) Vgl. Staatsarchiv Ludwigsburg, E 170 b // Bü 39; Hundert Jahre Staatliche Gewerbeförderung in Württemberg. 1848 - 1948, Stuttgart 1948; Landesgewerbeamt Baden-Württemberg. Das Landesgewerbeamt stellt sich vor, Stuttgart 1973; Finke, Karl Konrad: Bestände der ehemaligen Bibliothek des Württembergischen Landesgewerbeamts in Stuttgart. In: Zukunft braucht Herkunft, Stuttgart 1997, S. 65-67.
Im Zuge der Industrialisierung wurde ein zunehmender Bedarf nach einer staatlichen Förderung von Gewerbe und Handel v.a. im Bereich der Standardisierung und des Eichwesens für Maße und Gewichte spürbar. Auf Bitten der Gesellschaft für die Beförderung der Gewerbe gründete König Wilhelm I. von Württemberg im Jahr 1848 die Königliche Centralstelle für Gewerbe und Handel. Das damit verbundene Musterlager wurde zum Grundstein für das spätere Landesgewerbemuseum. 1856 kam eine Sammlung von Patentschriften hinzu. Um durch vergleichende Studien technische bzw. künstlerische Innovationen anzuregen, sammelte der Gründungspräsident der Centralstelle, Ferdinand von Steinbeis (1807-1893), auch zahlreiche Materialien aus dem Ausland. Patente und Muster sollten verfügbar sein und zur Beratung von Erfindern beitragen. 1896 fand die Eröffnung des Königlich Württembergischen Landesgewerbemuseums statt. Die Centralstelle benannte man 1921 in Landesgewerbeamt Stuttgart um und die dazugehörige Bibliothek hieß ab 1930 Öffentliche Bibliothek für Technik, Kunst und Wirtschaft. Teile der Bibliothek (v.a. die ursprünglich als eigene Teilsammlung unterhaltene Kunstbibliothek) wurden während des Zweiten Weltkriegs ausgelagert und konnten dadurch gerettet werden.

1952 erfolgte die Fusion mit dem Landesgewerbeamt Karlsruhe. Das Wirtschaftsministerium gliederte die Sammelgebiete Kunst und Technik aus seinem Zuständigkeitsbereich aus, was 1970 zur Auflösung der Landesgewerbeamtsbibliothek in ihrer bis dahin bestehenden Form führte. Im gleichen Gebäude (heute "Haus der Wirtschaft") blieben bis zur Verwaltungsreform von 2004 eine „Design-Bücherei" und eine „Bibliothek für berufliche Fortbildung" (einschließlich der Patent- und Normschriften). In die WLB gelangten alle nicht in dieses Sammelprofil passende Bände, darunter auch der wertvolle Altbestand, teilweise in späteren Nachlieferungen. ${ }^{1}$ 
88,5\% der aus dem Landesgewerbeamt übernommenen und eingearbeiteten Titel sind in deutscher Sprache verfasst. Unter den sonstigen europäischen Sprachen sind v.a. 647 französische und 400 englische Monographien hervorzuheben. Bei 9.498 Titeln $(67,6 \%)$ handelt es sich um Oktavbände, davon fast zur Hälfte schmale Kapselschriften. Nur etwa 8\% dieser Titel weist ein Erscheinungsjahr bis 1920 auf. Je größer das Format ist, desto höher liegt der Anteil älterer Titel: 3.115 Quart-Bände (32,8\%, bis 1920: 27,8\%), 815 Folio-Bände $(8,6 \%$, bis 1920: 74,1\%), 624 bisher katalogisierte Großfolio-Bände (6,6\%, bis 1920: 96\%). Dies lässt darauf schließen, dass im Laufe der Zeit anstelle überwiegend aus großformatigem Bildmaterial bestehender Bände eine andere Art von Literatur getreten ist, d.h. stärker textorientierte und weniger stark illustrierte sowie handlichere Sekundärliteratur. Die Beratungs- und allgemeine Fortbildungsfunktion für Gewerbetreibende wurde wichtiger als die didaktische Rezeption exemplarischer bzw. als Muster und zur Veranschaulichung von Standards fungierender Illustrationen. Titel aus den 1960er Jahren deuten darauf hin, dass es anders als in der Zeit bis zum 1. Weltkrieg im Gewerbe mehr um inhaltlichtheoretisches Detailwissen und um Steigerung von Effektivität und Qualität als um intuitiv-innovative Impulse ging. Da findet man eine Handwerkerfibel „für Meisterprüfung, Geschäftsgründung und Betriebsführung" (A22/3634), ein Buch über das bessere Wirtschaften durch bessere Liquidität (A22C/2326), ein Werk über "Physikalische Grundlagen der Formänderungsfestigkeit der Metalle" (A22Ca/729) bzw. zu metallischen Spezialwerkstoffen (A22a/2603) sowie ohne Illustrationen auskommende Dissertationen der Technischen Universität Karlsruhe (z.B. A22C/1696). Bücher mit zahlreichen Illustrationen gehören allerdings auch zum neueren Bestand, v.a. im Bereich der Archäologie (z.B. $A 22 a / 638)$ und der Kunst- und Kulturgeschichte (z.B. Reiseführer: A22/2516).

Ein besonders wertvolles Bestandssegment stellen die ca. 1.700 Großfolio-Bände (A22g) dar, von denen aus Kapazitätsgründen erst gut 600 Bände katalogisiert werden konnten. Über 500 dieser Titel sind digital verfügbar. Die Katalogisierung und Digitalisierung weiterer Einzelbände aus diesem Segment ist im Gang.

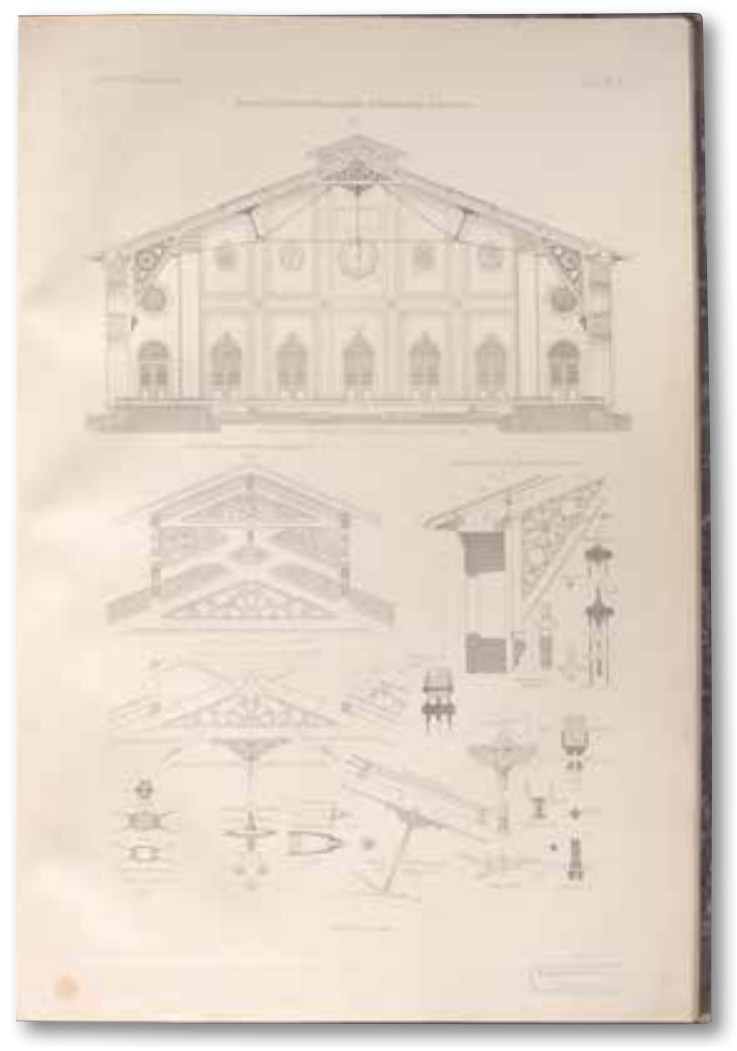

Abb. 2: Eisenkonstruktionen im Bauwesen (A22g/529)

Unter den Großformaten befinden sich auch Bände im Querformat sowie zahlreiche Mappenwerke mit lose eingelegten Bildtafeln. Bei den Illustrationstechniken trifft man auf eine große Vielfalt. Neben vereinzelt auftretenden Holzschnitten und Kupferstichen überwiegen Lithographien sowie Bildbände mit historischen Fotografien bzw. Lichtdrucken. Einige Beispiele veranschaulichen Wert und Bedeutung dieses Sammlungsteils.

Während bei den kleineren Formaten die meisten Bände zum Themengebiet Architektur an die UB Stuttgart abgegeben wurden, befinden sich unter den Großformaten noch viele Tafelwerke zu diesem Bereich. In gewisser Weise kann man dazu Bände mit Illustrationen zu architektonisch relevanten Gebäuden bestimmter Orte rechnen, etwa zu Potsdam (A22g/412) oder Rothenburg ob der Tauber (A22g/393). Dem entspricht Bildmaterial zur Außenund vor allem Innenarchitektur einzelner Gebäude oder Gebäudetypen. So bietet ein Band einen Überblick zum Typus Haus des Bürgertums (A22g/402) bzw. zum amerikanischen Herrenhaus (A22g/479). Ein Werk präsentiert Gebäude, die von einem bestimmten Architekten in Verona erbaut wurden (A22g/332). Der Raumzuschnitt und die Innenausstattung von Palästen lässt sich gut anhand entspre- 


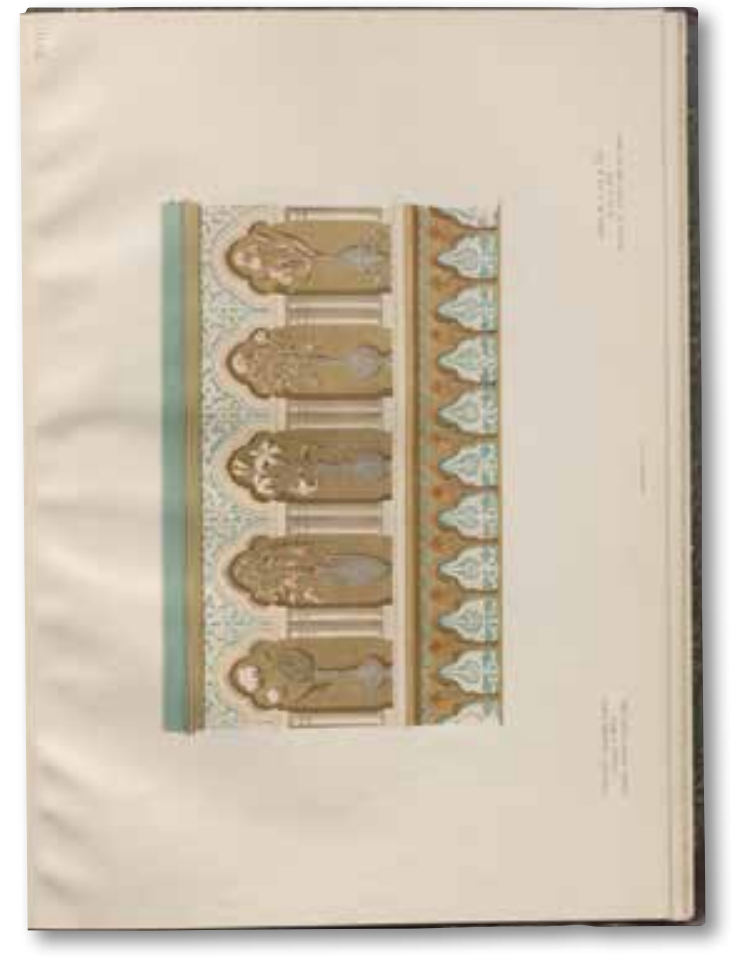

Abb. 3: Byzantinische Ornamente (A22g/381)

chender Beispiele vergleichen (z.B. A22g/392: Hôtel Lauzun, Paris; A22g/357: Schloss Nymphenburg, München; A22g/482: Schloss Fontainebleau). Außerdem findet man Illustrations-Sammlungen zur dekorativen Ausmalung von Innenräumen allgemein (z.B. A22g/466) sowie zur italienischen Innenarchitektur allgemein (z.B. A22g/496). Aus künstlerischer wie handwerklicher Sicht von Interesse sind Bildbände, die sich mit Einzelelementen von Architektur bzw. Bauwesen beschäftigen. Das gilt z.B. für schmiedeeiserne Schaufensterkonstruktionen (A22g/273), Plafonds italienischer Paläste (A22g/262), Fenster und Gardinen (A22g/291), Türen in Piemont (A22g/352) oder Eisenkonstruktionen im Hochbau (A22g/529).

Das Nebeneinander allgemeinerer und nach Gattung, Region bzw. Stil und Zeit spezifizierender Literatur kennzeichnet auch den Bestand zu anderen Themen. So begegnen Bände zur Ornamentik allgemein (z.B. A22g/584) wie zu enger definierten Anwendungsfällen des Ornaments als Verzierungsmotiv: z.B. Ornamente in der Architektur allgemein (A22g/324); Ornamente in der Architektur von Byzanz, Georgien und Russland (A22g/381) bzw. Italien im 15. Jahrhundert (A22g/525); ungarische Ornamente (A22b/6); ornamentale Decken- und Wandmalerei (A22b/805); Ornamente in Glasfenstern von Kirchen (A22b/771); Pflanzenornamente (A22g/569).

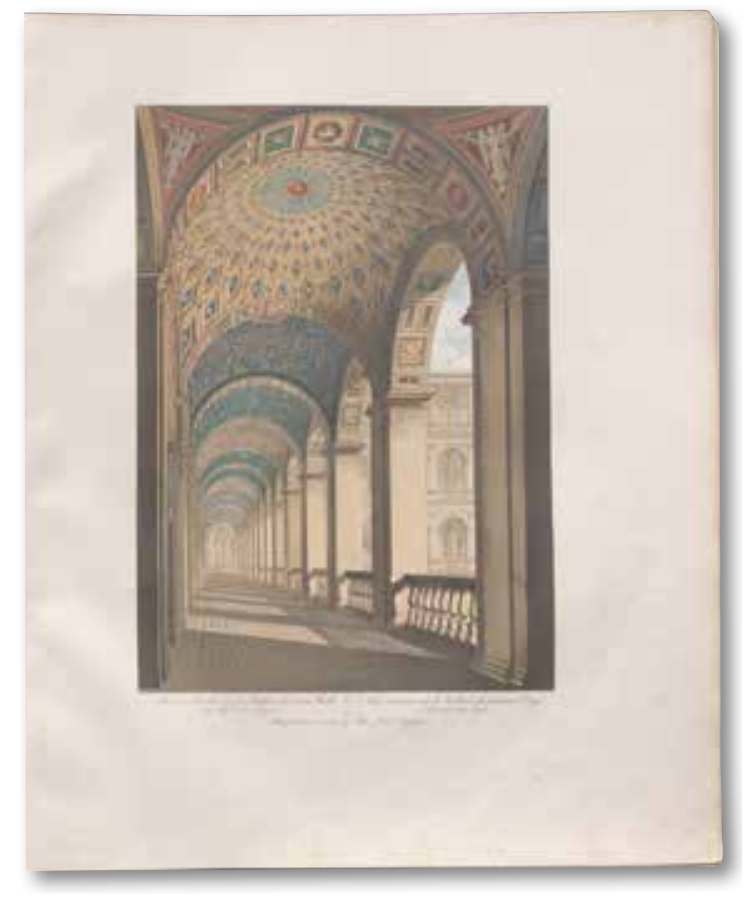

Abb. 4: Freskomalerei und Stuck in Italien (A22g/570)

Ähnliches gilt für das Themenfeld Malerei. In der Landesgewerbeamtsbibliothek wurden Monographien über Malerei und Reliefkunst allgemein (z.B. A22b/749) gesammelt, ebenso - schon etwas spezieller - zur Glasmalerei allgemein (A22g/383). Dann gibt es aber auch Bücher zur Blume als Motiv der Malerei (A22g/265), über dekorative Malerei in Frankreich im 11. bis 16. Jh. (A22b/823), orientalische Genrebilder (A22g/519), Freskomalerei und Stuck in italienischen Schlössern und Kirchen (A22g/570) sowie die Wandmalerei in der Wartburg (A22b/744).

Die Sammlung umfasst illustrierte Bände über das altorientalische Kunsthandwerk (A22b/2), aber auch zu Einzelobjekten des Kunsthandwerks wie z.B. Kandelaber (A22g/533), Leuchter und Vasen (A22g/543), Orient-Teppiche (A22g/415). Ein besonderes Interesse gilt den Möbeln und zwar in allen Stilarten (A22b/766), in moderner Gestaltung (A22b/740), als Kirchenmöbel (A22g/264), mit Schnitzereien (A22g/271) oder als dekorierte Stoffmöbel der napoleonischen Zeit (A22g/249).

Die Aufgabe der Unterstützung von Aus- und Fortbildung im ökonomischen Bereich erfüllte das Landesgewerbeamt auch dadurch, dass Muster und Vorlagen-Sammlungen für Berufe bestimmter Branchen zur Verfügung gestellt wurden. Dies 


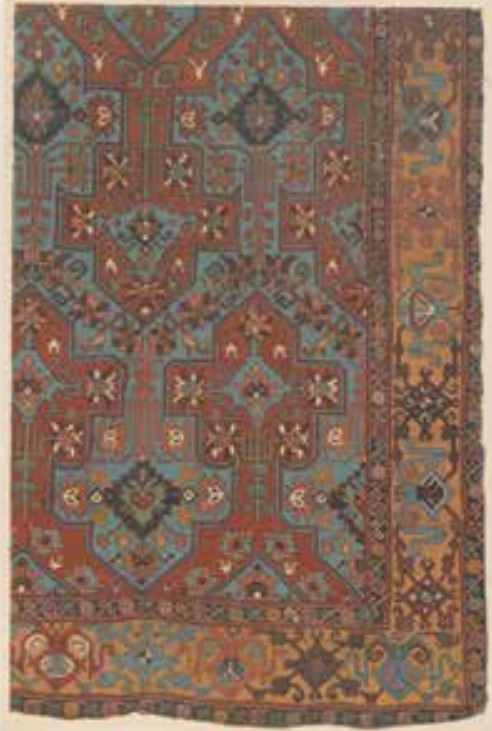

Abb. 5: Orientteppiche (A22g/415)

erklärt die relativ starke Präsenz modellhaften Bildmaterials für Handwerker, z.B. Maurer und Steinmetze (A22g/527), Tischler und Glaser (A22g/546), Schmiede (A22g/256), Maler (A22g/523) und Möbelschreiner (A22g/546; A22b/656).

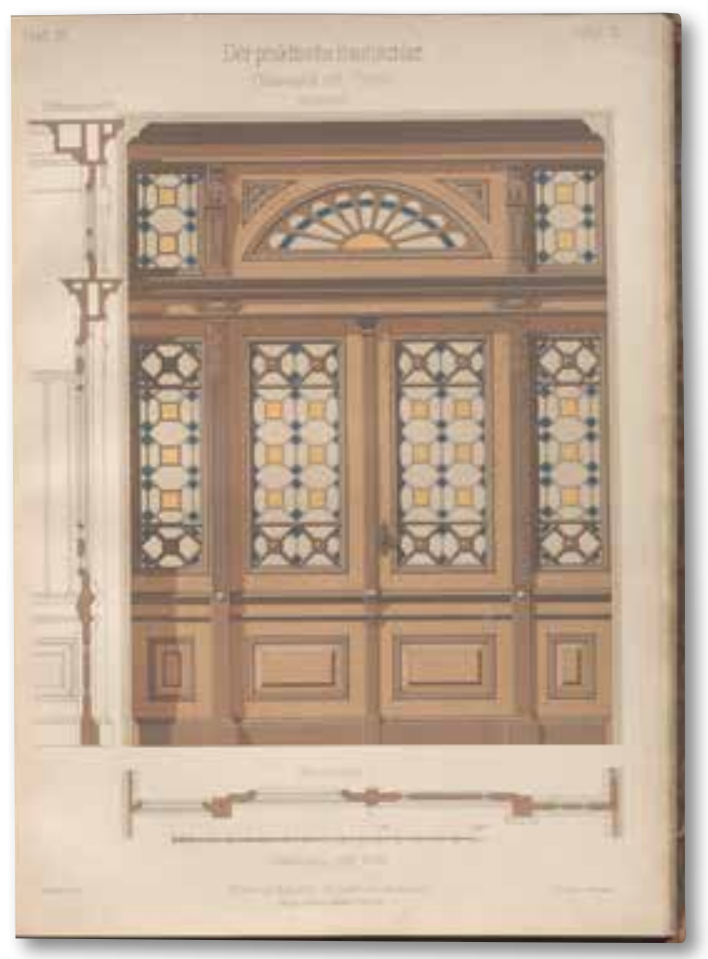

Abb. 6: Musterband für Schreiner (A22g/546)

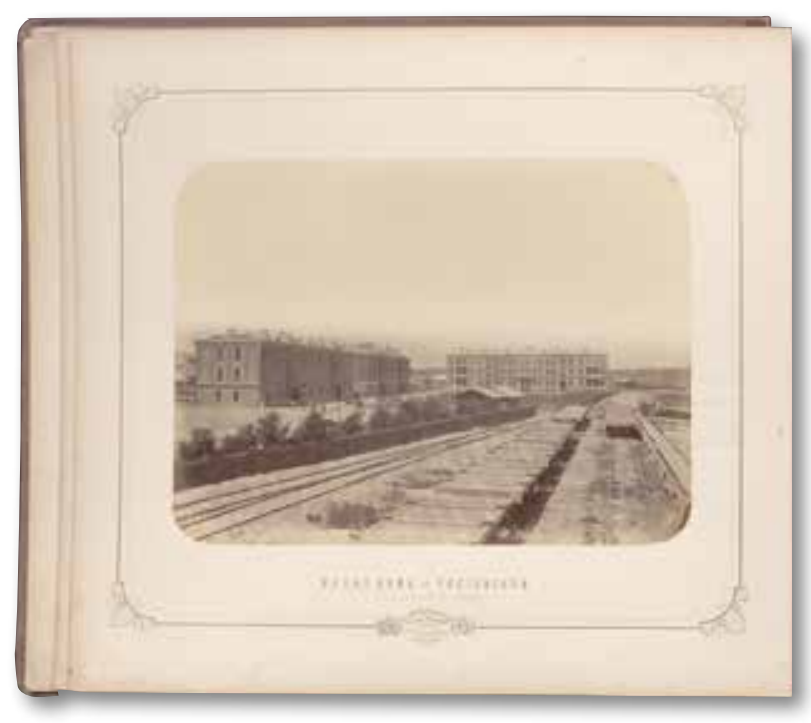

Abb. 7: Russische Eisenbahnstrecke (A22g/330)

Die Aufmachung und Zielgruppe ist ganz anders strukturiert bei Bänden von eher kulturgeschichtlicher Bedeutung. Dazu kann man Sammlungen historischer Fotografien etwa zu archäologischen Funden auf der Sinaihalbinsel (A22g/547) oder zu einer bestimmten russischen Eisenbahnstrecke (A22g/330) rechnen. Auch ein sechsbändiges Werk mit Abbildungen der Altertümer-Sammlung von Zar Nikolaus I. (1796-1855) zählt hierzu (A22g/317 u.a.).

Die Liste der Beispiele und Literaturtypen ließe sich fortsetzen, z.B. mit Nachdrucken älterer Werke (Wappenbuch: A22g/539) oder mit botanischen Tafelwerken (A22g/541). Der buchgeschichtliche Wert insbesondere der älteren, großformatigen Bände liegt auf der Hand. Die Bände aus dem Landesgewerbeamt insgesamt stellen einen konkreten Haftpunkt für die Rekonstruktion der deutschen Wirtschaftsgeschichte mit ihren Wechselwirkungen und internationalen Verflechtungen dar.

\section{Christian Herrmann}

